\title{
Non-Invasive Method of Measuring Blood Sugar in the Early Stage and Diagnose the Result Using Discrete Wavelet Transform
}

\author{
N.D Bobby, N.Prabhakaran², J.Thameema Begum², M.MuniReddy ${ }^{2}$ \\ ${ }^{1}$ Professor, ${ }^{2}$ Asst Professor, No 42 Alamathi road, Department of ECE, Vel Tech High Tech Dr Rangarajan \\ Dr Sakunthala Engineering College, Avadi Chennai 62.India
}

\begin{abstract}
Monitoring of blood glucose levels is clinically crucial in managing diseases affecting insulin secretion and resistance, most notably diabetes mellitus and cystic fibrosis. Blood glucose s used to monitor the amount of glucose in the blood, especially patients with symptoms with the abnormally of high or low blood glucose levels. Most recently, they enable diabetic patients to administer appropriate insulin doses to human health. The availability of in-house glucometers, as opposed to clinical-use equipment, has dramatically improved the quality of life of diabetic's patient. However, such monitors require a blood draw through finger pricks for each test, which causes pain and inconvenience. Each test also needs a new test-strip, contributing to the recurring cost of such a device. Typically the blood glucose monitoring is the non-invasive technique which may cause distress and discomfort, particularly in the pediatric patient. We introduce an architecture that uses Near Infrared (NIR) spectroscopy to determine blood glucose levels based on transmittance spectroscopy on the nose lobe. The Measurement of glucose in the respiratory fluid by a collection of exhaled breath in condensate and therefore, a potentially clinically useful method of estimating blood glucose levels identified and therefore it is the excellent agreement between these diabetics blood glucose level.
\end{abstract}

Keyword: Insulin secretion, Diabetes mellitus Glucometer, Near-infrared spectroscopy, Diabetes mellitus.

\section{Introduction}

Diabetes mellitus is a disease resulting from insufficiency of insulin in the body causing elevated blood-glucose levels known as hyperglycemia, or reduced glucose concentrations, known as hypoglycemia [1]. Insulin is the hormone secreted from the pancreas to mediate metabolic reactions from glucose level into the body. Initiates have taken to decrease blood glucose level in the body, and hence reduce the human risk to live a long life. Diabetes as associated with many medical conditions such as celiac disease, cystic fibrosis, tuberculosis and heart disease for all groups of peoples ${ }^{[2]}$. Such conditions result in retinopathy which leads to blindness, nephropathy rise to renal failure, and peripheral nerve affected with increased in the human risk of foot ulcer, cardiovascular diseases or final stage of cancer which leads to death. Diabetes mellitus is the manageable disorder in which the of blood glucose is monitored daily so that diabetics affected person won't get afraid because every time the affected person feels the blood glucose level will rise faster, it leads to significant risk ${ }^{[3]}$. The diagnosis and monitoring of blood glucose in the human body currently used in medical studies such as pre-diabetes patients involves the use of blood tests before and after fasting in real time situation. Blood glucose level is done by drawing the blood samples from the patient using a typical glucometer to measure the reading in digital form. This glucometer measurement involves pricking a drop of blood from the human body and placing the drop of blood on a sensitive area of the strip which is inserted in an electronic reading instrument in digital form ${ }^{[4]}$. This method is very painful, invasive and it is unsafe for diabetic's patient. Therefore, it will not suit all people, especially where the patient wants several samples of a blood test each day. The emerging technique built on the advancement measurement technologies provides a noninvasive method for diabetic's disease and diagnosis of the disease in the early stage itself ${ }^{[5]}$. The application of acetone detection has potential attention which 
rapidly distinguishes between healthy and unhealthy diabetic patients. These remarkable results are achieved to improve analytical techniques performed in the last ten years ${ }^{[6]}$. This method uses the single instrument and skilled operators for testing the blood glucose level both in fasting and after fasting with the duration of minimum two hours ${ }^{[7]}$. Furthermore, the technique includes low cost with high accuracy of result for all type of diabetics' patient, i.e., TYPES I, TYPE II diabetics ${ }^{[8]}$. The next section wills briefly summaries about the materials and methods and findings which are used to identify the earlier stage of diabetics.

\section{Material and Method}

Envoy Kirchsteiger, Luca Zachariah, Eric Reynard, Luigi del has proposed the issue of online adjustment and recalibration for nonstop checking the blood glucose level to severe diabetics in the beginning time ${ }^{[9]}$. Two distinctive parametric relations among interstitial and blood glucose are examined, and valuable calculations to adaptively appraise the parameters inside those relations are proposed ${ }^{[10]}$. One trademark is the clear thought of estimation vulnerability of the gadget used to gather the alignment estimations. Another component is the programmer discovery of finger stick estimations that are not appropriate to be utilised for alignment. Since the techniques depend on the arrangement of straight grid imbalances bringing about arched improvement issues, the calculations are of moderate computational multifaceted nature and could be actualized on a CGM gadget. The strategies were surveyed on clinical information from 17 diabetic patients, and the enhancements as for the present best in class appear ${ }^{[11]}$. Yang Jin; Hong Wang, Hayman Cal, Zhenjiang Lv, Shiyuan Yang, Hayman Cai, Jun Feng Jiang and Tsinghua has proposed the electrochemical techniques which have been generally utilised in the concoction and pharmaceutical ventures, which require exact fixation estimations, substance response location and examination for people with diabetes quiet. The electrochemical potentiostat, the central component in electrochemical instruments, have been examined as an exciting issue tending to the trouble of applying highaccuracy consistent voltage and Pico ampere current estimations ${ }^{[12]}$. Then, dependable potentiometer is very favourite for convoluted mechanical situations with commotions just as prerequisites of remote screens. This paper portrays a potentiostat for automatic glucose estimation that isn't only exact yet also blame tolerant to ensure consistently high quality in modern conditions.
They showed adaptable $\mathrm{pH}$ sensor and glucose biosensor altered by attractive dabs and graphene was proposed. The ruthenium dioxide detecting films were kept by radio recurrence sputtering framework, and the screen printed system was utilised to build the silver directing wires and a protective layer of the cathodes. To improve execution of the $\mathrm{pH}$ sensor and glucose biosensor, the smaller scale fluidic gadget had been used and created ${ }^{[13]}$. In the estimation forms, the different $\mathrm{pH}$ and glucose arrangements were researched in different stream rates. As indicated by the test results, the natural affectability of the $\mathrm{pH}$ sensor was improved, and the natural affectability of the 2/grapheme/attractive dot GOx-Nafion glucose biosensor was upgraded from 10.628 to $13.541 \mathrm{mV} / \mathrm{mm}$ Concerning the remote detecting estimations, the remote detecting framework which agreed the ZigBee standard was utilised to transmit the signs of the $\mathrm{pH}$ or glucose estimations in this examination. Xbee gadget, Arduino Mega 2560, a readout circuit, $\mathrm{pH}$ or glucose biosensor and PC. Without diabetes, your body tracks glucose levels throughout the day and night to guarantee the appropriate measure of insulin is discharged at the correct time with hazard free appraisal. To effectively oversee diabetes, an observing framework is expected to check your glucose levels in the body reliably. The breathing sensors incorporate transducers connected to the human body. For instance, customarily utilised strategy utilises two versatile groups implanted with sensors around the ribcage and stomach area to screen their developments. Data on the different movements of the ribcage and midriff give data about breath exertion. Albeit worthy for shorter timeframes, this strategy is inadmissible for long haul checking or observing of dozing patients. A fasting blood glucose test is the favoured strategy to analyse diabetes and guideline out different conditions. This test is done after an individual has had nothing to eat or drink except water for somewhere around 8 hours. It is for the most part begun medium-term so the test should be possible in the first part of the day. A self-observing test or blood glucose checking by finger prick is a quick and straightforward technique for distinguishing issues with glucose levels. The average blood glucose levels fluctuate contingent upon which test was performed, regardless of whether an individual was fasting before the test, and whether any exceptional dietary or glucose substances were given amid testing. Expanded dimensions of blood glucose, a condition known as hyperglycemia, might be brought about by the accompanying blood glucose level in a reasonable state. 


\section{Findings}

The figure 1 shows the hardware circuit and to implemented to the patient for monitoring the blood glucose level with fast fasting. The net results can be achieved in digital a value which is the accurate measurement and it can vary from +20 to -20 variations in the blood sugar level. The figure 2 shows the hardware circuit and to implement to the patient health monitoring the blood glucose level without fasting. The net results can be achieved in digital a value which is the accurate measurement and it can vary from +20 to -20 variations in the blood sugar level. Both the hardware circuit implemented for patient health monitoring is the non invasive method of testing the blood glucose level. The figure 3 and 4 shows the output interns of dyadic wavelet transformation and the time domain factor and probability distribution of signal analyzed with respect to discrete wavelet transform.

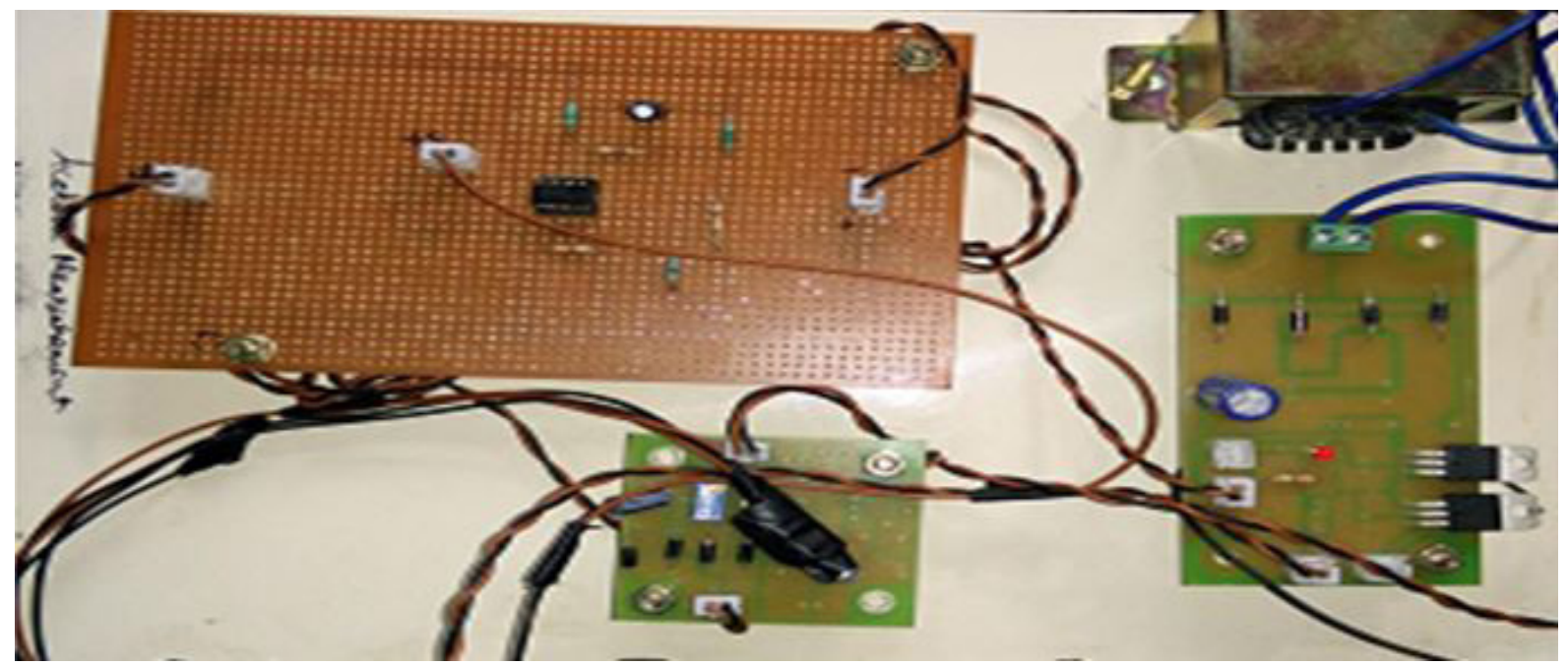

Figure 1 Before Fasting

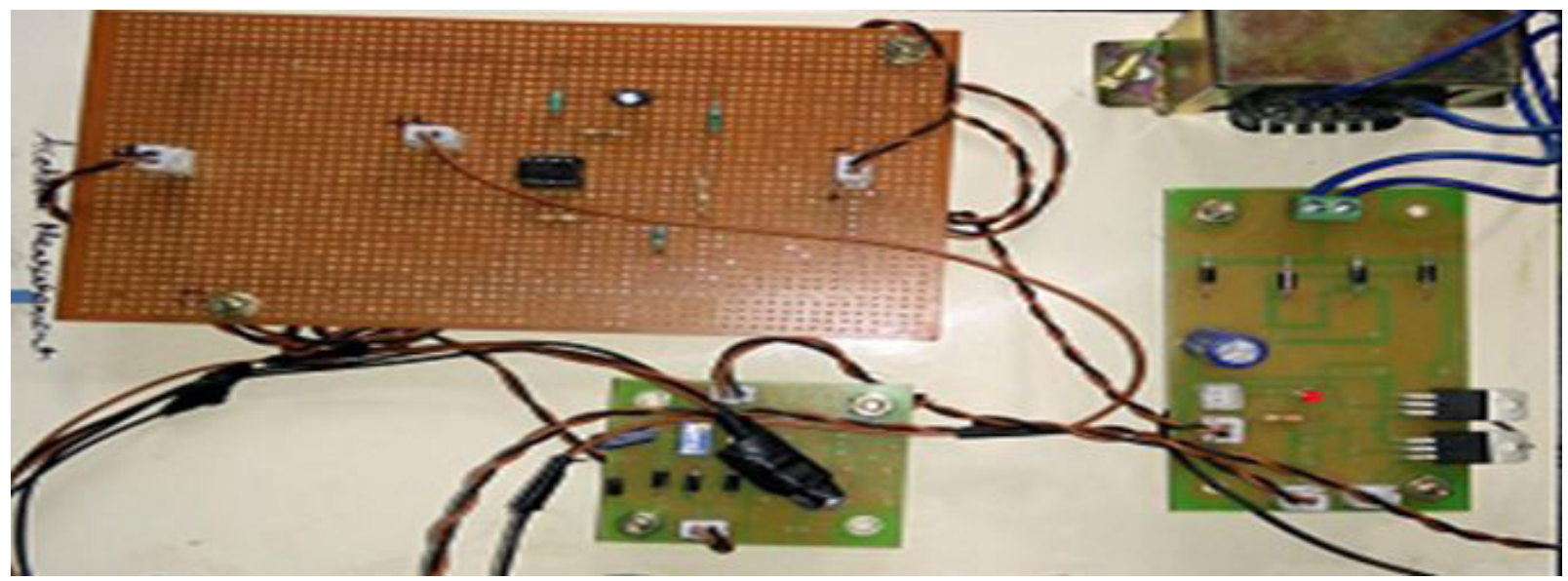

Figure 2: After fasting 


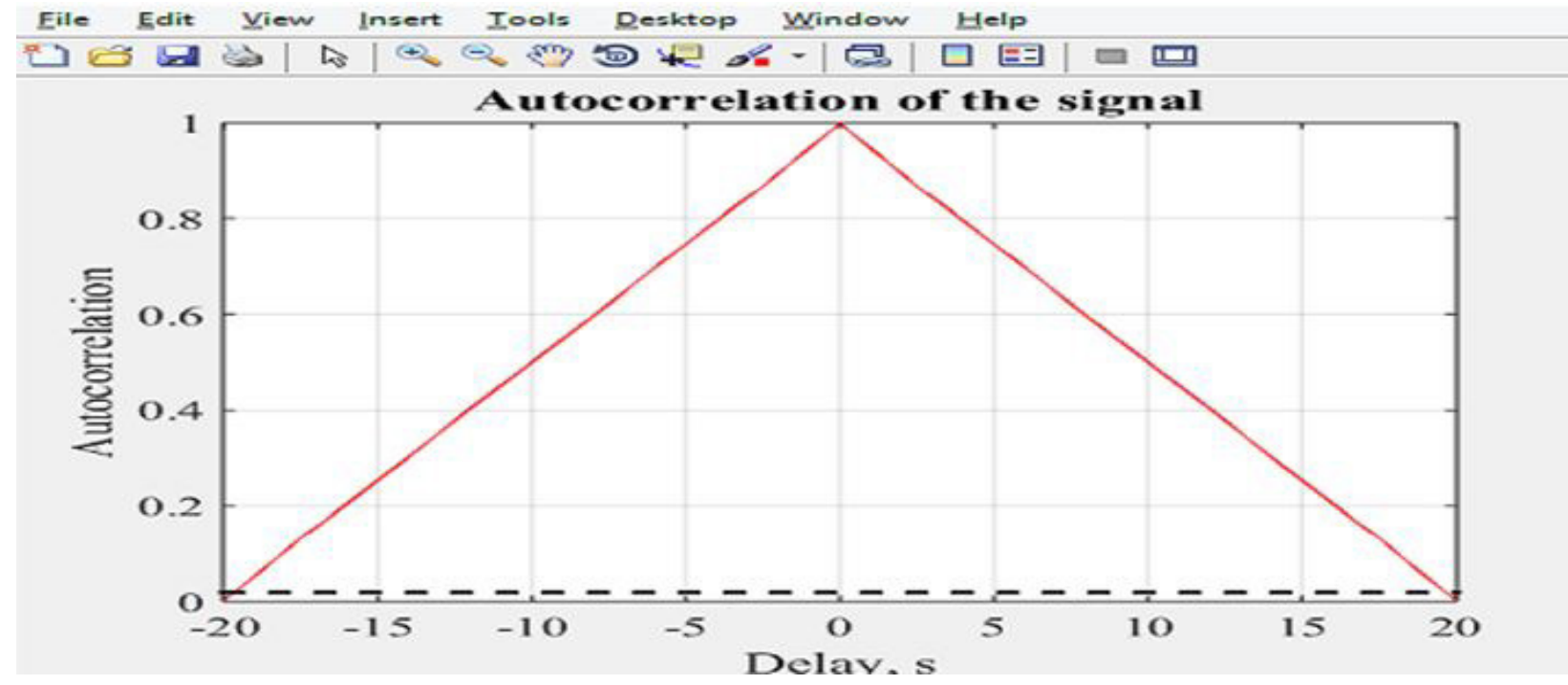

Figure 3: Simulated Output

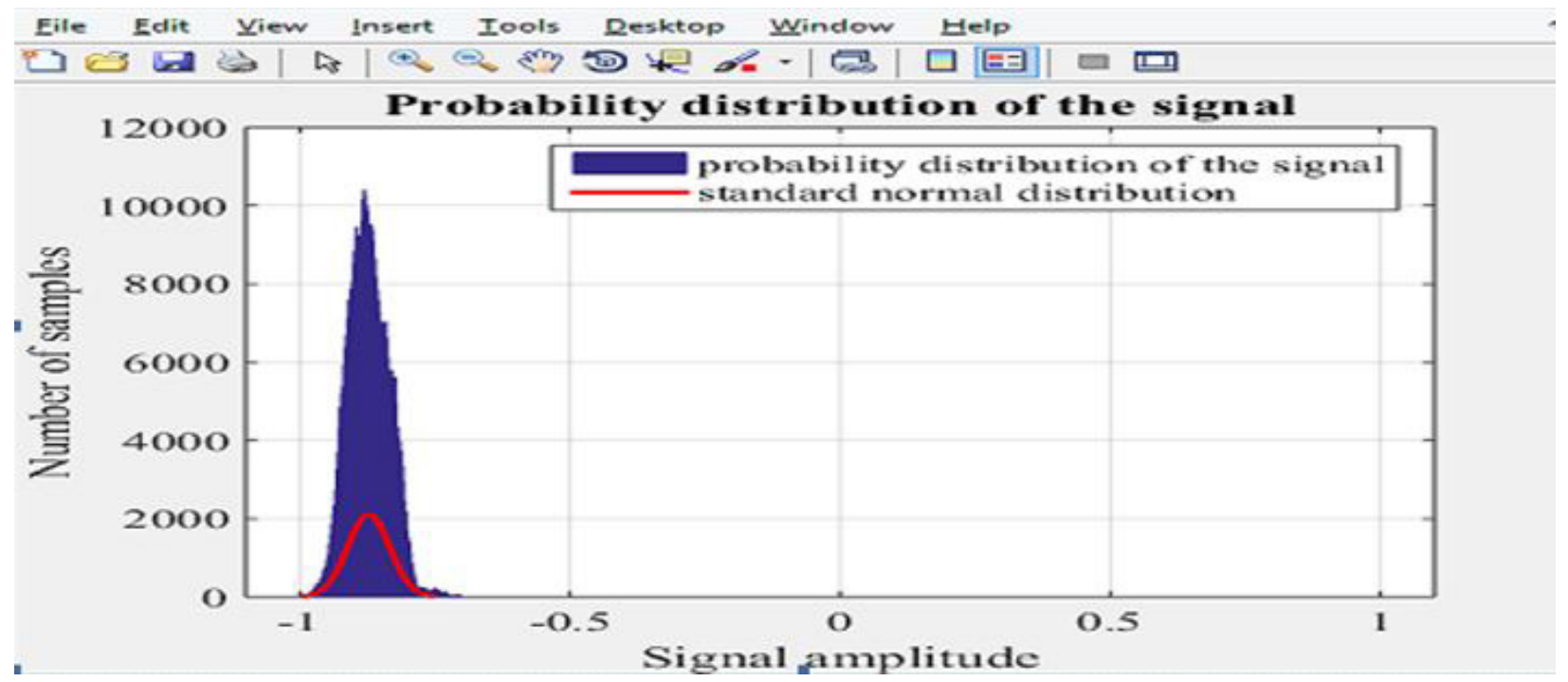

Figure4: Probability distribution of a signal

\section{Conclusion}

There have been continuous advances in the field of glucose monitoring during the last four decades, which have led to the development of highly evolved blood glucose meters, non-invasive glucose monitoring (NGM) devices and continuous glucose monitoring systems (CGMS).Glucose monitoring is an integral part of diabetes management, and the maintenance of physiological blood glucose concentration is the only way for a diabetic to avoid life-threatening diabetic complications. This technique is highly useful for random test in the blood glucose level for monitoring the health condition in the early stage and probably when diagnosis is identified in the early stage the condition to maintain blood sugar in Normal Mode.

\section{Future Developments:}

In future, the glucose level can be measured by using sweat, saliva and tears. Monitoring of blood glucose level can be measured by using noninvasive techniques. Noninvasive techniques are used than invasive technique which can create distress and discomfort such as finger prick method. Glucose monitoring is an integral part of diabetes management, and the maintenance of physiological blood glucose concentration is the only way for a diabetic to avoid life-threatening diabetic complications. 
Conflict of Interest: There is no conflict of interest regarding this research paper

\section{Source of Funding: Self}

Ethical Clearance: The research work proves in novelty of the proposed work

\section{References}

1. Developments R. Glucose Sensing for Diabetes Monitoring: Recent Developments. sensor. 2017;1-21.

2. Saasa V, Malwela T, Beukes M, Mokgotho M, Liu C, Mwakikunga B. Sensing Technologies for Detection of Acetone in Human Breath for Diabetes Diagnosis and Monitoring. diagnostics. 2018;(Dm):1-17.

3. Wang Z, Wang C. Is breath acetone a biomarker of diabetes? A historical review on breath acetone measurements. J Breath Res. 2013;037109(7):118.

4. Righettoni M, Tricoli A. Toward portable breath acetone analysis for diabetes detection. J Breath Res. 2011;037109:1-9.

5. Agarwal D, Bansal A. Non-invasive techniques for the screening of diabetic retinopathy . J Biomed bio Eng. 2017;1(2):25-30.

6. Id ASM, Covington JA, Arasaradnam RP, Id SE, Connell NO, Kyrou I, et al. An improved machine learning pipeline for urinary volatiles disease detection : Diagnosing diabetes. PLoS One. 2018;22(sep):1-20.

7. Ashok V, Kumar N. Determination of Blood Glucose Concentration by Using Wavelet Transform and Neural Networks. iran J Med Sci. 2013;38(1):51-6.

8. Developments R. Glucose Sensing for Diabetes Monitoring: Recent Developments. sensor. 2017;1866(17):1-21.

9. Geng $\mathrm{Z}$, Tang $\mathrm{F}$, Ding $\mathrm{Y}$, Li $\mathrm{S}$, Wang $\mathrm{X}$. Noninvasive Continuous Glucose Monitoring Using a Multisensor- Based Glucometer and Time Series Analysis. Sci Rep [Internet]. Springer US; 2017;7(May):1-10. Available from: http://dx.doi. org/10.1038/s41598-017-13018-7

10. Id ASM, Covington JA, Arasaradnam RP, Id SE, Connell NO, Kyrou I, et al. An improved machine learning pipeline for urinary volatiles disease detection : Diagnosing diabetes. PLoS One. 2018;22(sep):1-20.

11. Iyer A, Jeyalatha S, Sumbaly R. D IAGNOSIS OF D IABETES U SING. Int J data Min Knowl Manag Process. 2015;5(1):1-14.

12. Narkhede P, Dhalwar S, Karthikeyan B. NIR Based Non-Invasive Blood Glucose Measurement. Indian J Sci Technol. 2017;9(41)(November 2016):1-8.

13. Singh $P$, Kaur H, Singh KVP. Non-invasive Blood Glucose Level Measurement from LASER Reflected Spectral Patterns Images. iosr J Eng. 2013;3(8):6-10. 